\title{
Photoacoustic Measurement of Electron Injection Efficiencies and Energies from Excited Sensitizer Dyes into Nanocrystalline $\mathrm{TiO}_{2}$ Films
}

\author{
Carlos Serpa, ${ }^{*, \dagger}$ Johann Schabauer, ${ }^{\ddagger}$ Ana P. Piedade,$^{\S}$ Carlos J. P. Monteiro, ${ }^{\dagger}$ Mariette M. Pereira ${ }^{\dagger}$ \\ Peter Douglas, ${ }^{\ddagger}$ Hugh D. Burrows, ${ }^{\dagger}$ and Luis G. Arnaut ${ }^{*, \dagger}$ \\ Chemistry Department, University of Coimbra, P3004-535 Coimbra, Portugal, Chemistry Department and School of \\ Engineering, Swansea University, Swansea, SA2 8PP, United Kingdom, and CEMUC-Mechanical Engineering \\ Department, University of Coimbra, P3030-788 Coimbra, Portugal
}

Received March 14, 2008; E-mail: serpasoa@ci.uc.pt

Solar cells with a dye adsorbed on nanocrystalline $\mathrm{TiO}_{2}$ can attain light-to-electrical energy conversion efficiencies of $10 \%,{ }^{1}$ and raise hopes for clean, economical solar energy conversion. The first chemical step following light absorption involves injection of an electron from a dye excited-state into the $\mathrm{TiO}_{2}$ conduction band. The efficiency and energy of electron injection are of primary importance for the overall efficiency of these solar cells. However, direct measurement of these efficiencies and energies has remained elusive. The quantum yield of electron injection is often estimated indirectly either from the excited-state lifetime in the absence of injection and the electron injection rate constant or from transient absorption spectroscopy, which is complicated by both overlap between ground-state and transient absorptions and short transient lifetimes. The energy for electron transfer is usually estimated from the redox potential of the electronically excited dye and the semiconductor conduction band energy. However, this is ill defined for nanocrystalline semiconductors. ${ }^{2}$ We describe a new approach, based on detection of the pressure wave generated upon conversion of excitation energy into heat, as the electronically excited dye evolves to a charge-separated (CS) state with the electron located in the conduction band. This is the basis of photoacoustic calorimetry (PAC). We report heterogeneous electron-injection efficiencies and energies ( $2 \%$ and $0.05 \mathrm{eV}$ precision, respectively) using this technique.

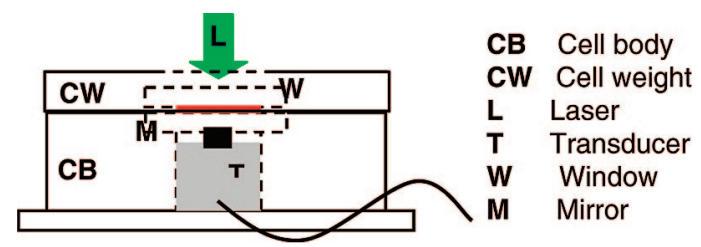

Figure 1. Front-face PAC cell for solid/liquid samples. The unfocused laser beam diameter matches that of the transducer. A thin film of liquid (glycerol or hexadecane) wets the space between the $\mathrm{TiO}_{2}$ surface and the dielectric mirror.

Time-resolved PAC measures energies and quantum yields of photochemical intermediates in solution. ${ }^{3-6}$ PAC is based on the deconvolution of the signal of a sample with that of a photoacoustic reference, defined as a dye that converts all the electronic energy absorbed into heat on a time scale much shorter than the timeresolution of acoustic detection. Attempts to extend this to the study of similar processes in thin films are confronted with the need to design PAC cells with both high sensitivity and high reproducibility, in particular when sample and reference are interchanged. ${ }^{7,8} \mathrm{~A}$ cell

Chemistry Department, University of Coimbra.

* Swansea University.

\& CEMUC-Mechanical Engineering Department, University of Coimbra. that meets this challenge is shown in Figure 1. The sample (a dye adsorbed on nanocrystalline $\mathrm{TiO}_{2}$ deposited on a glass slide) is placed on a dielectric mirror previously covered with a thin layer of acoustic coupling medium (hexadecane or glycerol), while a quartz window with the same pretreatment is placed on the top of the sample. A 2.25 MHz Panametrics transducer (model 5676) is coupled to the back of the mirror, and the sample holder ensures that laser beam, transducer, mirror, sample, and window are always in the same relative position. The cell also includes a $1.5 \mathrm{~kg}$ weight to exert a constant pressure on top of the window. This is critical to achieve the desired reproducibility. Samples are irradiated through the quartz window, following the front-face PAC cell design which is known to provide maximum sensitivity. ${ }^{3,6} \mathrm{We}$ employed the second harmonic of Nd:YAG lasers (EKSPLA PL 2143A or Spectra

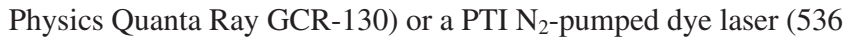
$\mathrm{nm}$ ) for sample excitation. The PAC signals were not influenced by either laser pulse width ( $30 \mathrm{ps}$ to $8 \mathrm{~ns}$ ) or repetition frequency ( 2 to $10 \mathrm{~Hz}$ ). Procedures for data collection and analysis were the same as for solution time-resolved PAC. ${ }^{5,6}$

Energy balance requires that the energy, corrected for the relative yields, of the laser pulse $\left(E_{\mathrm{h} v}\right)$ minus that released in formation of the transient charge-separated state $\left(\phi_{1} E_{\mathrm{h} v}\right)$ and that lost radiatively $\left(\Phi_{\mathrm{F}} E_{\nu \max }\right)$, equals that of the CS state

$$
\Phi_{\mathrm{CS}} E_{\mathrm{CS}}=\left(1-\phi_{1}\right) E_{h v}-\Phi_{\mathrm{F}} E_{\nu \max }
$$

where $E_{\nu \max }$ is the energy at the maximum emission intensity, and $E_{\mathrm{h} v}=2.33 \mathrm{eV}$ at $532 \mathrm{~nm}$. The value of $\phi_{1}$ is obtained from deconvolution of the reference and sample signals. When the rate of formation of the CS state is orders of magnitude faster than its decay, high-frequency transducers do not respond to the slow decay and the ratio of amplitudes between reference and sample waves gives essentially the same results as deconvolution of slow and fast events (see Supporting Information). The implicit assumption in eq 1 is that the pressure wave comes exclusively from thermal processes, that is, volume changes are negligible. The validity of this depends on the chemistry of the system.

The outstanding performance of $\mathrm{Ru}(\mathrm{II})(\mathrm{dcbpy})_{2}(\mathrm{NCS})_{2}(\mathrm{~N} 3$, dcbpy $=4,4^{\prime}$-dicarboxy-2,2'-bipyridine) adsorbed on nanocrystalline $\mathrm{TiO}_{2}$ and the detailed data available for this system,,${ }^{2,10,11}$ motivated its choice for the first measurements of PAC at solid/solution interfaces. The reference and sample signals must be collected following irradiation under the same conditions. MnTPP is a good photoacoustic reference in solution, ${ }^{6}$ but it does not adsorb onto $\mathrm{TiO}_{2}$. Tetraphenylporphyrin (TPP) was synthesized via the nitrobenzene method, ${ }^{12}$ chorosulfonated, complexed with $\mathrm{Mn}^{3+}$ and hydrolyzed to produce Mn(III)TPPS, which is a good photoacoustic reference for $\mathrm{TiO}_{2}$ films. Similar experiments were made with the corresponding copper complex. Details on the synthesis, charac- 


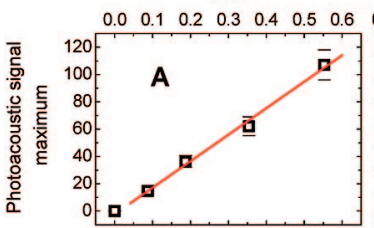

$20 \quad 40 \quad 60 \quad 80 \quad 100 \quad 0350$
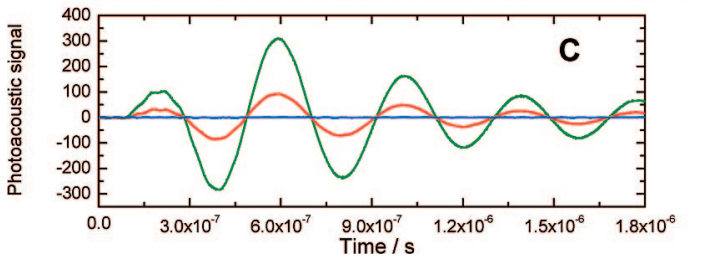

Figure 2. (A) Intensity dependence of PAC waves on the fraction of energy absorbed for MnTPPS adsorbed on $\mathrm{TiO}_{2}$; five independent measurements were made for each absorbance. (B) Fraction of the fast nonradiative decay of N3 at various laser intensities. (C) PAC waves of MnTPPS (green) or $\mathrm{N} 3$ (red) adsorbed on $\mathrm{TiO}_{2}$, normalized for the absorbance of 0.18 at 532 $\mathrm{nm}$; the blue line is the signal with $\mathrm{TiO}_{2}$ and no dye; all data with glycerol.

terization, and preparation of $\mathrm{TiO}_{2}$ films using the squeegee print method are given as Supporting Information. Neither of these porphyrins showed emission in aqueous solution or when adsorbed to $\mathrm{TiO}_{2}$ films, and no transient absorptions were seen on timescales of tens of nanoseconds.

Porphyrins with sulfonate anchoring groups take longer to adsorb to titania surfaces than the N3 dye with its carboxylate groups. PAC is best performed with samples having absorptions $0.2-0.3$ at the excitation wavelength, after subtraction of the $\mathrm{TiO}_{2}$ background signal. These absorbances were obtained by immersing slides in ethanolic solutions for 5 min for N3 or for a few hours for MnTPPS samples. Under our experimental conditions no fluorescence was detected from the N3 samples. The acoustic coupling between all parts of the cell significantly influences the amplitude and the onset of the acoustic wave. The constant weight exerted on the quartz window standardizes the acoustic coupling. Coupling with the dielectric mirror and quartz window was further ensured by the thin layer of liquid (glycerol or hexadecane) applied on the back and front of the slide. Figure 2 presents PAC waves and their reproducibility. For the same absorbances, the waves of CuTPPS and MnTPPS are similar, both acting as calorimetric references. The layer of liquid on top of the slide probably additionally stabilizes the charge separated state to different extents, depending on polarity. As glycerol has a dielectric constant comparable to ethanol it provides similar stabilization of the chargeseparated state $\left(\Delta G_{\mathrm{CS}}{ }^{\mathrm{O}}=1.75 \mathrm{eV}\right) .{ }^{11}$

The fraction of heat released promptly by $\mathrm{N} 3$, measured by the ratio of the PAC waves of MnTPPS and N3, is $\phi_{1}=0.25 \pm 0.02$ in glycerol and $\phi_{1}=0.45 \pm 0.02$ in hexadecane. Equation 1 with $E_{\mathrm{CS}}=1.75 \mathrm{eV}$, gives $\Phi_{\mathrm{CS}}=0.99 \pm 0.03$ for glycerol, where the uncertainty comes mostly from imperfect compensation between volume and entropy changes, ${ }^{5}$ discussed in more detail below. The value obtained by transient spectroscopy measurements is $0.80 \pm$ $0.15,{ }^{13}$ which is probably too low in view of the rates involved.

Equation 1 also gives reaction energies when the CS efficiencies are known. The fact that there is hardly any medium dependence on this interfacial ET rate ${ }^{10}$ and that the ET barrier is dominated by high-frequency modes, ${ }^{11}$ implies that the efficiency of electron injection in the semiconductor is not appreciably dependent on the polarity of the medium. Using $\Phi_{\mathrm{CS}}=0.99$ with $\phi_{1}=0.45 \pm 0.02$ measured with hexadecane, we obtain $E_{\mathrm{CS}}=1.3 \mathrm{eV}$. This implies that the energy of oxidized $\mathrm{N} 3$ relative to the $\mathrm{TiO}_{2}$ conduction band appears to increase by $0.45 \mathrm{eV}$ when the solvent is changed from an alcohol to a hydrocarbon, Figure 3, meaning that the energy of
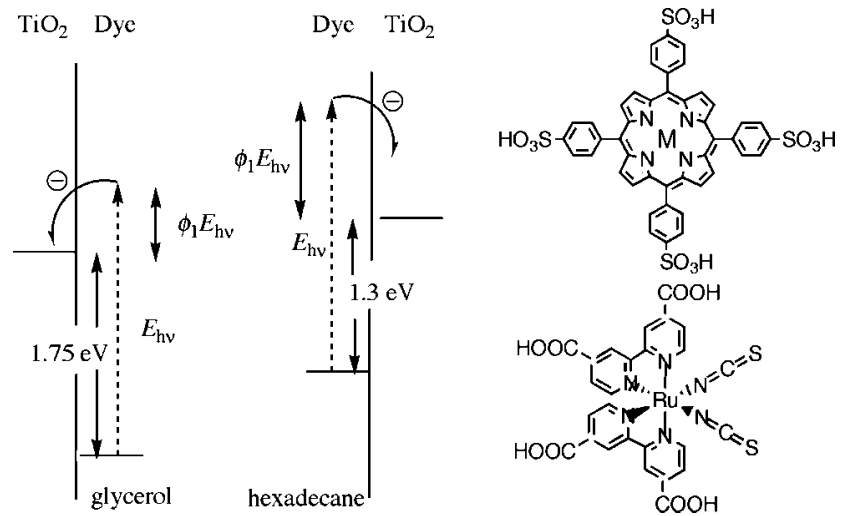

Figure 3. Schematic diagram showing the relative energies of the transients involved in the electron injection from dyes in $\mathrm{TiO}_{2}$. The absolute energies in hexadecane are not known.

the hole located in the dye is more sensitive to solvent effects. TRPAC is unique for determination of the energy of such chargeseparated states and, when the oxidation potential of the dye is known, it also gives the energy of the edge of the semiconductor conduction band. We believe that this can provide unique insight into the nature of the conduction band.

Our analysis neglects volume and entropy changes. Indeed, the structural volume changes in the oxidation of N3 are minimal, the electrostriction entropy changes compensate electrostriction volume changes, ${ }^{5}$ and the translational entropy of the electron in the conduction band is small (see Supporting Information), leading to $\Phi_{\mathrm{CS}} \Delta G_{\mathrm{CS}}{ }^{0} \approx\left(1-\phi_{1}\right) E_{\mathrm{h} v}$ for these systems.

Finally, it is worth mentioning that the extension of this technique to operational solar cells and to the study of other solid/liquid interfaces is straightforward and its adaptation to biological systems can also be envisaged.

Acknowledgment. Financial support from FEDER and FCT (project POCI/QUI/55505/2004) and grants from FCT SFRH/BPD/ 27139/2006 (A.P.P.), SFRH/BD/37652/2007 (C.J.P.M.), and EPSRC through a DTA grant to J.S. are acknowledged.

Supporting Information Available: Synthesis and characterization of MnTPPS and CuTPPS, preparation of $\mathrm{TiO}_{2}$ films, PAC cell details, electronic spectra, PAC waves, and entropy estimation. This material is available free of charge via the Internet at http://pubs.acs.org.

\section{References}

(1) Nazeeruddin, M. K.; Kay, A.; Rodicio, I.; Humphry-Baker, R.; Miller, E.; Liska, P.; Vlachopoulos, N.; Gratzel, M. J. Am. Chem. Soc. 1993, 115, 6382-6390.

(2) Kuciauskas, D.; Freund, M. S.; Gray, H. B.; Winkler, J. R.; Lewis, N. S. J. Phys. Chem. B 2001, 105, 392-403

(3) Arnaut, L. G.; Caldwell, R. A.; Elbert, J. E.; Melton, L. A. Rev. Sci. Instrum. 1992, 63, 5381-5389.

(4) Braslavsky, S. E.; Heibel, G. E. Chem. Rev. 1992, 92, 1381-1410.

(5) Serpa, C.; Arnaut, L. G. J. Phys. Chem. A 2000, 104, 11075-11086.

(6) Pineiro, M.; Carvalho, A. L.; Pereira, M. M.; Gonsalves, A. M. d. A. R.; Arnaut, L. G.; Formosinho, S. J. Chem.-Eur. J. 1998, 4, 2299-2307.

(7) Katalnikiv, I. V.; Auweraer, M. v. d.; Schryver, F. C. d. J. Photochem. Photobiol. A 1994, 77, 103-107.

(8) Nonell, S.; Marti, C.; Garcia-Moreno, I.; Costela, Á.; Sastre, R Appl. Phys. $B$ 2001, 72, 355-360.

(9) Serpa, C.; Gomes, P. J. S.; Arnaut, L. G.; Formosinho, S. J.; Pina, J.; Melo, J. S. d. Chem.-Eur. J. 2006, 12, 5014-5023.

(10) Hannappel, T.; Burfeindt, B.; Storck, W.; Willig, F. J. Phys. Chem. B 1997, 101, 6799-6802.

(11) Gratzel, M.; Moser, J.-E. In Electron Transfer in Chemistry; Balzani, V., Gould, I. R. , Eds.; Wiley-VCH: New York, 2001; Vol. 5, pp 589-644..

(12) Gonsalves, A. M. d. A. R.; Varejao, J. M. T. B.; Pereira, M. M. J. Heterocycl. Chem. 1991, 28, 635-640.

(13) Katoh, R.; Furube, A.; Yoshihara, T.; Hara, K.; Fujihashi, G.; Takano, S.; Murata, S.; Arakawa, H.; Tachiya, M. J. Phys. Chem. B 2004, 108, 4818-4822.

JA801928Z 